\title{
Guiding Graph Exploration by Combining Layouts and Reorderings
}

\author{
Michael Burch, Kiet Bennema ten Brinke, Adrien Castella, Ghassen Karray \\ Sebastiaan Peters, Vasil Shteriyanov, Rinse Vlasvinkel \\ Eindhoven University of Technology \\ Eindhoven, The Netherlands
}
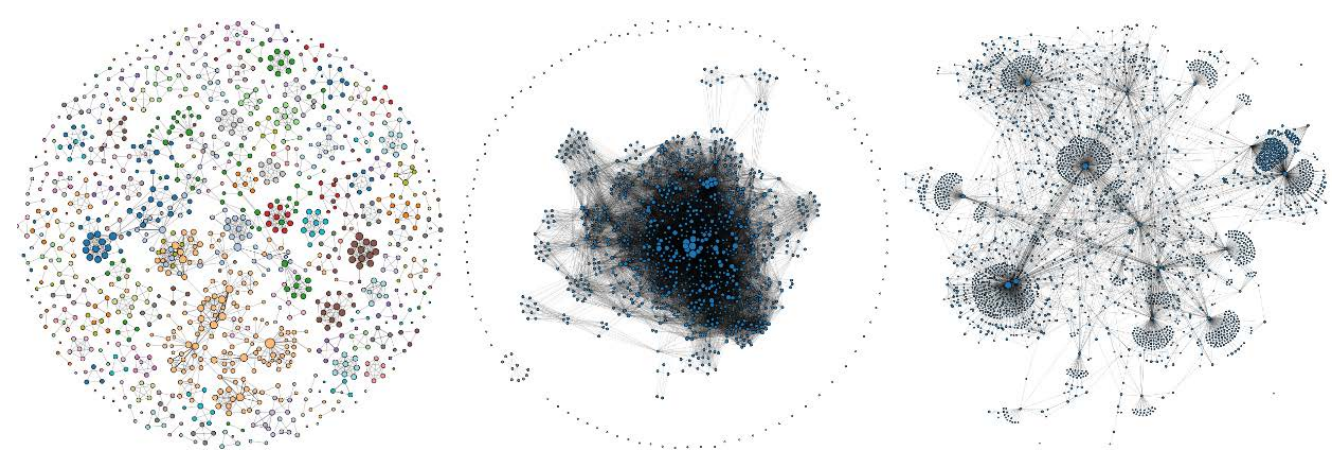

Figure 1: Node-link diagrams of real-world datasets: co-authorships, co-citations, and a CPAN distribution [cpa 2019].

\begin{abstract}
Visualizing graphs is a challenging task due to the various properties of the underlying relational data. For sparse and small graphs the perceptually most efficient way are node-link diagrams whereas for dense graphs with attached data, adjacency matrices might be the better choice. Since graphs can contain both properties, being globally sparse and locally dense, a combination of several visualizations is beneficial. In this paper we describe a visually and algorithmically scalable approach to provide views and perspectives about graphs as interactively linked node-link as well as adjacency matrix visualizations. The novelty of the technique is that insights like clusters or anomalies from one or several combined views can be used to influence the layout or reordering of the others. Moreover, the importance of nodes and node groups can be detected, computed, and visualized by taking into account several layout and reordering properties in combination as well as different edge properties for the same set of nodes. We illustrate the usefulness of our tool by applying it to graph datasets like co-authorships, co-citations, and a CPAN distribution.
\end{abstract}

\section{CCS CONCEPTS}

- Human-centered computing $\rightarrow$ Graph drawings.

Permission to make digital or hard copies of all or part of this work for personal or classroom use is granted without fee provided that copies are not made or distributed for profit or commercial advantage and that copies bear this notice and the full citation on the first page. Copyrights for components of this work owned by others than ACM must be honored. Abstracting with credit is permitted. To copy otherwise, or republish, to post on servers or to redistribute to lists, requires prior specific permission and/or a fee. Request permissions from permissions@acm.org.

VINCI 2020, December 8-10, 2020, Eindhoven, The Netherlands

(C) 2020 Association for Computing Machinery.

ACM ISBN 978-1-4503-8750-7/20/12 . .\$15.00

https://doi.org/10.1145/3430036.3430064

\section{KEYWORDS}

Graph visualization, Node-link diagrams, Adjacency matrices, Layouts, Reorderings

\section{ACM Reference Format:}

Michael Burch, Kiet Bennema ten Brinke, Adrien Castella, Ghassen Karray and Sebastiaan Peters, Vasil Shteriyanov, Rinse Vlasvinkel. 2020. Guiding Graph Exploration by Combining Layouts and Reorderings. In The 13th International Symposium on Visual Information Communication and Interaction (VINCI 2020), December 8-10, 2020, Eindhoven, The Netherlands. ACM, New York, NY, USA, 5 pages. https://doi.org/10.1145/3430036.3430064

\section{INTRODUCTION}

Graph data occurs in various application fields like call dependencies in software engineering [Burch et al. 2012], friendship relations in social networks [Henry and Fekete 2006, 2007; Henry et al. 2007], areas of interest connections in eye tracking data [Burch et al. 2014], or traffic situations in road networks [Greilich et al. 2009].

Exploring such data requires advanced visual metaphors, in the best case interactively linking several of those to benefit from the positive effects of all of them. For example, node-link diagrams [Di Battista et al. 1999] are useful for small and sparse graphs (see Figure 1) whereas adjacency matrices [Elmqvist et al. 2008] are best for large and dense networks [Ghoniem et al. 2005; Okoe et al. 2019]. However, using only one concept may lead to degradations of performance at some task [Rosenholtz et al. 2005].

To give even more ways to find insights than just one fixed visualization technique we provide several node-link layouts [Soni et al. 2018] as well as several matrix reordering techniques [Behrisch et al. 2016]. The node-link diagrams follow aesthetic graph drawing criteria [Purchase 1997] while the adjacency matrices support the finding of different grouping and clustering patterns depending on the user tasks and which reordering strategy is requested. All of the 
views and parameter adaptations can be selected on users' demand but the tool can also automatically suggest one, based on graph data properties. Our novel linked visualization strategy provides ways to adapt a view based on insights from other views, for example clusters found in the adjacency matrix can be used to guide the layout of the node-link diagram and vice-versa.

Our novel technique allows interactively selecting clusters in several views and letting the algorithm compute the intersection of node sets. The output is a filtered version of the graph that contains all nodes that occur for all requested properties. This gives a hint about the importance of a node or node group based on algorithmic concepts like layouts and reorderings but additionally enhanced by visual depictions of the output of such algorithms in combination.

\section{RELATED WORK}

Graphs have been introduced [Euler 1741] for finding a solution to the graph-theoretic problem known as the 'Seven Bridges of Königsberg'. To visually illustrate the problem Euler used the visual metaphor of node-link diagrams [Di Battista et al. 1999]. Several years ago they were pretty powerful due to the fact that the graph data was small and sparse, with only a few vertices and edges.

In these days, graphs are typically huge and preprocessing and managing graph data is already a big challenge [Eades and Klein 2018]. However, visualizing the relational data [von Landesberger et al. 2011] as a node-link diagram in a naive way leads to visual clutter [Rosenholtz et al. 2005]. Even advanced layout algorithms [Kaufmann and Wagner 2001] can only partially solve this problem since the immense size and density of the relational data does not allow to follow individual outliers or anomalies. In most cases those are occluded by dense graph regions, for example clusters of nodes.

Adjacency matrices are powerful visual concepts [Elmqvist et al. 2008]. They allow thousands of vertices to be represented and all the weighted and directed edges in-between. However, adjacency matrices show problems when following paths, which is on the other hand also problematic for node-link diagrams if the graphs exceed a certain size [Ghoniem et al. 2005; Keller et al. 2006]. Moreover, an unordered adjacency matrix will not show any structure and hence, matrix reordering strategies must be supported [Behrisch et al. 2016], also guided by a human user [Behrisch et al. 2020].

Hybrid representations use the benefits of both concepts [Henry and Fekete 2007; Henry et al. 2007], however, following a direct integration of both concepts does not support several independent layouts and reorderings, at least it becomes difficult. On the other hand such a combination might hide insights that might be seen in the node-link diagrams or adjacency matrices when represented separately. Consequently, we follow the original approach by Henry and Fekete [Henry and Fekete 2006]. Although their approach is already equipped with various features we further add the concept of exploiting visual properties from formerly laid out and reordered node-link diagrams and adjacency matrices to select and filter nodes.

In this paper we focus on displaying a graph in several views supporting the human observer by interactively changing the views, layouts, and reorderings on demand, inspired by a multiple visual metaphors approach [Burch 2015, 2017]. For this reason, we take into account insights from the views and parameter configurations while also linking and highlighting the insights.

\section{DATA AND VISUALIZATIONS}

We describe a data model for graphs, how a layout and a reordering can be generated and which processes and algorithms are involved, as well as how visual graph properties can be combined and linked to influence other views and graph visualizations.

\subsection{Graph Data}

A graph $G=(V, E)$ typically consists of a finite number of vertices $V:=\left\{v_{1}, \ldots, v_{n}\right\}$ and a finite number of edges $E:=\left\{e_{1}, \ldots, e_{m}\right\} \subseteq$ $V \times V$. The edges might be weighted, meaning a weight function is attached, mapping each edge to a real-valued number, $f: E \longrightarrow \mathbb{R}$.

\subsection{Layouts and Reorderings}

A layout of a graph $G$ is a positioning of the given vertices $V$ to $n$ (normally) distinct locations in the display space whereas the edges in-between are drawn as either straight, curved, orthogonal [Holten et al. 2011], or partial links [Bruckdorfer et al. 2017; Burch et al. 2011], depending on several aesthetic graph drawing criteria to be followed [Ware et al. 2002].

A reordering is applied to an adjacency matrix [Behrisch et al. 2016] following a certain property that produces a rearrangement of the vertices in a way that a certain (typically user-defined) property holds, for example, a cluster structure at the diagonal.

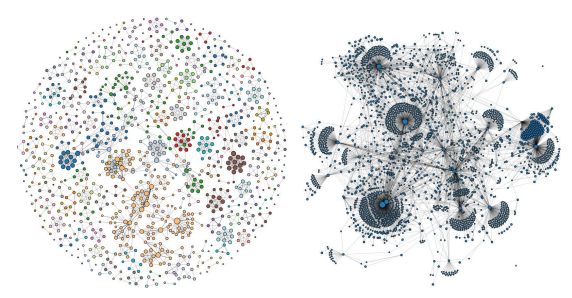

Figure 2: On the left: Force-directed node-link diagram of a coauthorship dataset with 1,053 nodes and 3,504 edges. On the right: Force-directed node-link diagram of the CPAN distributions dataset with 2,724 nodes and 7,669 edges.

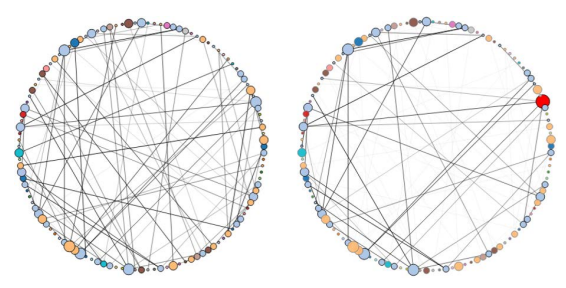

Figure 3: The radial layout of the node-link diagram of the randomly generated dataset - on the left is the initial radial layout and on the right is the filtered radial layout.

\subsection{Combining Graph Properties}

The novelty of the technique is that insights like clusters or anomalies from one view can be used to influence the layout or reordering of the other. Moreover, the importance of nodes and node groups can be detected, computed, and visualized by taking into account 


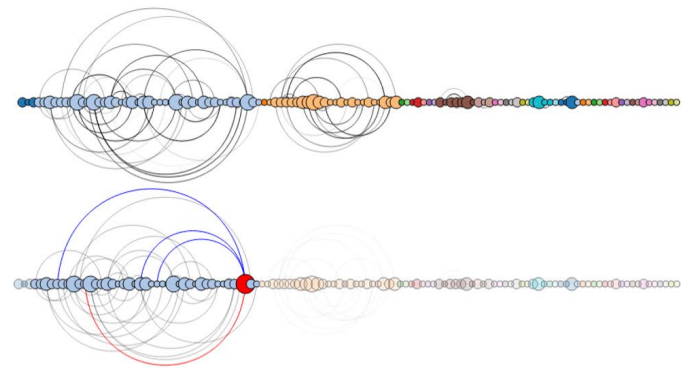

Figure 4: The arc layout of the node-link diagram of the randomly generated dataset - on top is the initial arc diagram and on the bottom is the filtered arc diagram.

several layout and reordering properties in combination. The first step towards this direction is that several node-link layouts and adjacency matrix reorderings of the same graph dataset are computed and then the visual properties of the produced diagrams are taken into account to build an intersection or union set of the nodes under investigation. This principle helps to identify certain node groups that belong together under different circumstances, meaning their relation among each other is stronger than if just one representation is taken into account.

The identification of clusters works in two stages: First, several algorithms are applied to a graph dataset and second, the visual outcomes of these algorithms are combined to detect strongly clustered nodes or nodes that do not fall in any of the clusters, which might be considered 'real' outliers. Such a feature is useful as a filtering function and has several benefits compared to the standard 'one visualization' filtering.

Moreover, we can apply an automatic node group detection based on node group densities. This can be done for both, the node-link layouts as well as the adjacency matrices. Although this is a useful approach, it is best if the human users are involved in the node detection process equipped with their perceptual abilities to detect visual patterns rapidly [Ware 2004].
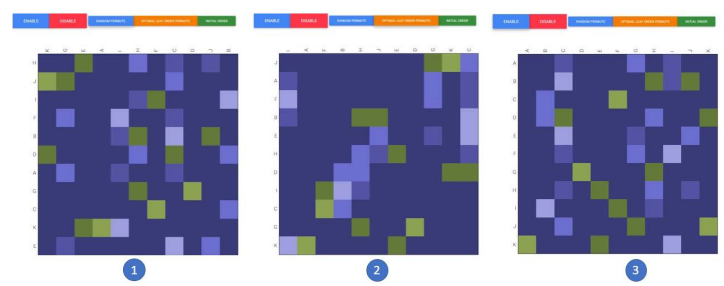

Figure 5: Three reorderings of the same graph.

\subsection{Node-Link Diagrams and Adjacency Matrices}

Our tool provides three layouts for a node-link diagram: a forcedirected [Fruchterman and Reingold 1991], a radial [Baur and Brandes 2007], and an arc layout [Greilich et al. 2009]. The force-directed layout presents the larger clusters close to the center and the smaller ones and single isolated nodes close to the border of the diagram (see Figure 2). This helps to better identify outliers than if those are located inside a hairball-like node-link diagram.
The radial layout displays the nodes aligned as a circle in which each node has an equal distance to the center and edges represented as straight lines connecting the nodes (see Figure 3 ). The radial layout is useful for seeing the density of a graph in local regions as well as the connections between nodes.

The arc layout aligns the nodes on a straight line, with edges represented as arcs connecting the nodes (see Figure 4). The arc layout has the advantage of being able to highlight components if the node order is optimized.

Currently, the adjacency matrix is displayed as a pixel map, where the links are shown as filled grid cells where the corresponding connected nodes meet. Each cell's color depends on the weight of the edge - edges with a higher weight are lighter compared to edges with a lower weight (with the default color scheme). In the tool, we implemented five reordering strategies, a random reordering and an option to go back to the original ordering (see Figure 5 for three reordering examples in a simple adjacency matrix visualization). Those are a weight sum order, a reverse Cuthill-McKee order, a shortest path order, a unique values order, a mean values order, and a random permutation order.

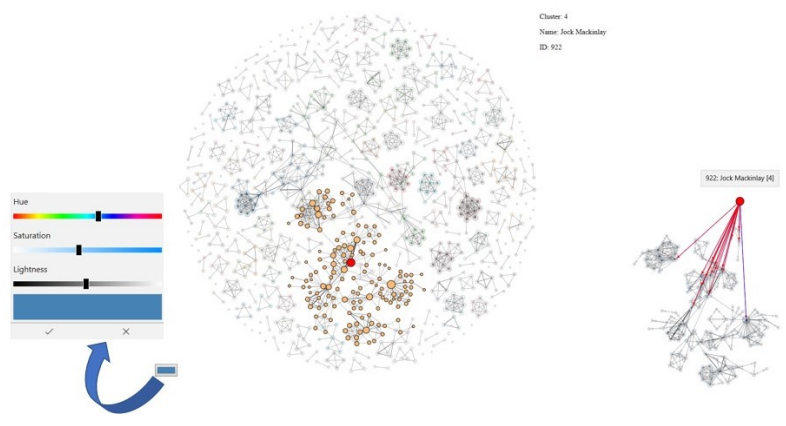

Figure 6: Selecting a cluster of nodes to take a closer look at details, for example, a node that is important in this cluster.

\section{GUIDING GRAPH EXPLORATION}

We illustrate the usefulness of our visualization tool by applying it to an author similarity dataset and show an adjacency matrix, with two datasets for the force-directed layout of the node-link diagram containing CPAN distributions and co-authorship data. It will also use a generated small dataset to showcase the radial and arc layout as a node-link diagram. Different datasets will be used because adjacency matrices with a high number of edges are more interesting to study, but the node-link diagram struggles with rendering graphs with a high number of edges and the radial and arc layouts are more useful for smaller datasets.

The co-authorship data includes 1,053 nodes and 3,504 edges. Once the users have uploaded the dataset, they can view it as a nodelink diagram. The force-directed layout of the node-link diagram displaying the co-authorship dataset is shown on the left in Figure 2. Users can see that bigger connected components are displayed in the center of the visualization, while smaller ones are displayed relatively closer to the border of the visualization and single isolated nodes are even closer to the border. Also, nodes with a larger amount of incoming edges appear bigger compared to others. By using 


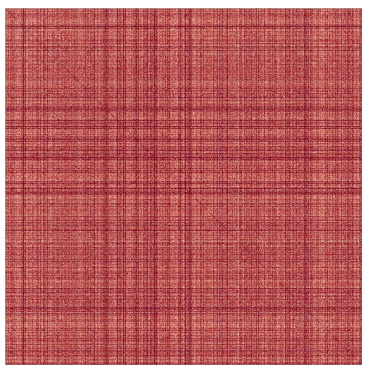

(a)

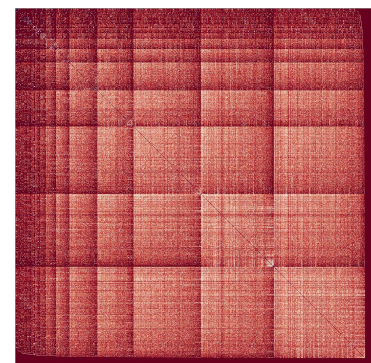

(b)
Figure 7: (a) The adjacency matrix of the initial authorsimilarity dataset with 1,053 nodes and 907,164 edges. (b) The reordered dataset using the reverse Cuthill-McKee algorithm.

this information it can be concluded that the single nodes can be considered as outliers and ignored, hence they do not flow into the selection of the most important and strongly connected nodes. Lastly, it can be observed that every connected component of the graph has its own color, making it easy for the user to differentiate between the different components of the graph. Selecting individual components from the node-link layouts can hence lead to useful insights that could not be found by the standard solutions (see Figure 6).
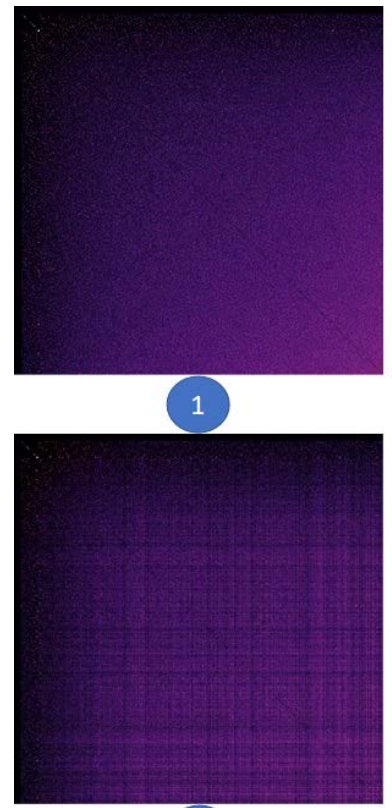

3

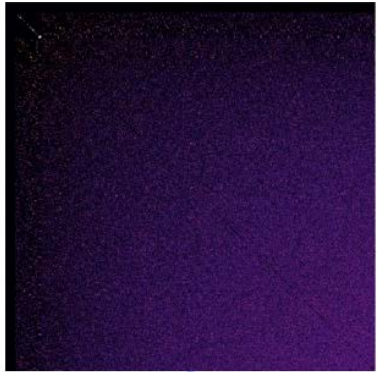

2
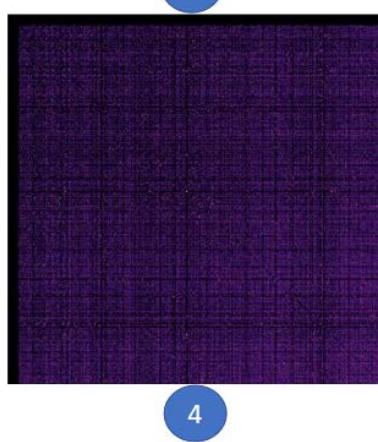

Figure 8: Matrices of the author-similarity dataset reordered using the mean values ordering (1), weight sum ordering (2), unique value ordering (3), and shortest path ordering (4).

The CPAN distributions dataset contains 2,724 nodes and 7,669 edges. The force-directed layout of the node-link diagram for this dataset can be seen on the right in Figure 2. When the graph is rendered, users can notice how most of the nodes in the graph are connected in a large connected component. Within it, there are a few nodes that appear larger, as they have a higher number of incoming edges. Also, these nodes appear to be surrounded by groups of smaller nodes with fewer edges. These nodes represent packages, on which other packages depend. They are also used more frequently, compared to others. Nodes that are not part of the large connected component can be interpreted as outliers and therefore be ignored.

The randomly generated dataset contains 120 nodes, the maximum weight of an edge is 30 and the chance of an edge is 0.007 (0.7 $\%)$. The radial layout for this dataset can be seen in Figure 3 and the arc layout in Figure 4. In Figure 3 the initial radial layout on the left shows the whole dataset. In the filtered radial layout on the right users can see that most of the edges belong to the component of blue colored nodes if they select a node from the component of blue nodes. The arc layout can help the user finding co-occurrences in the data. In Figure 4 on the left the initial layout reveals the connected components in the graph. The filtered layout on the right reveals connections of a selected node within its component.

The author-similarity data includes 1,053 nodes and 907,164 edges. The initial adjacency matrix displaying this dataset is shown in Figure 7 (a). In the initial order there are no clear patterns that can be observed. However, when the reverse Cuthill-McKee ordering is executed on the data, users can see symmetric diagonal block patterns. The reordered matrix can be seen in Figure 7 (b).

This means that there are strongly connected components and the nodes within them share similar characteristics. Users can also see that the components increase the size from the top left to the bottom right corner of the matrix, where the connected components are the biggest. Also, in each component, the edges are ordered by their weight from the bottom right corner of the component to the top left corner, where the weight of the edges is the biggest. In Figure 8 the adjacency matrix displays the same dataset using the mean values ordering (1), weight sum ordering (2), unique value ordering (3), and shortest path ordering (4).

Users can see the reordered matrices in (1), (2), and (3) ordering the edges by weight from the top left to the bottom right corner of the matrix, where the weight of the edges is the largest. The reordered matrices (3) and mostly (4) form block patterns, which also confirm that there are strongly connected components in the data.

\section{CONCLUSION AND FUTURE WORK}

In its current state, our web-based tool has achieved its goal of rendering beautiful visualizations, which could be used to detect patterns and insights from the data, combining several layouts and reorderings. It provides useful layouts, interactions and reordering strategies that help the user to achieve this. However, there are always aspects that could be improved to make our application even better. Currently, the tool can only work with datasets in a specific format, which is not user-friendly. To improve this, our application has to support all possible datasets or at least the most common ones. Although our tool works on touchscreen devices, there are issues related to panning, zooming, and selecting elements. It would be useful to extend the repertoire of reordering strategies for the adjacency matrix by implementing other complex approaches. 


\section{REFERENCES}

2019. Datasets. https://github.com/gephi/gephi/wiki/Datasets

Michael Baur and Ulrik Brandes. 2007. Multi-Circular Layout of Micro/Macro Graphs. In Proceedings of 15th International Symposium of Graph Drawing, GD. 255-267.

Michael Behrisch, Benjamin Bach, Nathalie Henry Riche, Tobias Schreck, and JeanDaniel Fekete. 2016. Matrix Reordering Methods for Table and Network Visualization. Computer Graphics Forum 35, 3 (2016), 693-716.

Michael Behrisch, Tobias Schreck, and Hanspeter Pfister. 2020. GUIRO: User-Guided Matrix Reordering. IEEE Transactions on Visualization and Computer Graphics 26 , 1 (2020), 184-194.

Till Bruckdorfer, Sabine Cornelsen, Carsten Gutwenger, Michael Kaufmann, Fabrizio Montecchiani, Martin Nöllenburg, and Alexander Wolff. 2017. Progress on Partial Edge Drawings. Journal of Graph Algorithms and Applications 21, 4 (2017), $757-$ 786.

Michael Burch. 2015. Dynamic Graph Visualization with Multiple Visual Metaphors. In Proceedings of the 8th International Symposium on Visual Information Communication and Interaction, VINCI. 27-34.

Michael Burch. 2017. The Dynamic Graph Wall: Visualizing Evolving Graphs with Multiple Visual Metaphors. Journal of Visualization 20, 3 (2017), 461-469.

Michael Burch, Fabian Beck, Michael Raschke, Tanja Blascheck, and Daniel Weiskopf. 2014. A Dynamic Graph Visualization Perspective on Eye Movement Data. In Proceedings of Symposium on Eye Tracking Research and Applications, ETRA. 151-158.

Michael Burch, Christoph Müller, Guido Reina, Hansjörg Schmauder, Miriam Greis, and Daniel Weiskopf. 2012. Visualizing Dynamic Call Graphs. In Proceedings of the Vision, Modeling, and Visualization Workshop. 207-214.

Michael Burch, Corinna Vehlow, Natalia Konevtsova, and Daniel Weiskopf. 2011 Evaluating Partially Drawn Links for Directed Graph Edges. In Proceedings of the 19th International Symposium on Graph Drawing, GD. 226-237.

Giuseppe Di Battista, Peter Eades, Roberto Tamassia, and Ioannis G. Tollis. 1999 Graph Drawing: Algorithms for the Visualization of Graphs. Prentice-Hall.

Peter Eades and Karsten Klein. 2018. Graph Visualization. In Graph Data Management Fundamental Issues and Recent Developments. 33-70.

Niklas Elmqvist, Thanh-Nghi Do, Howard Goodell, Nathalie Henry, and Jean-Daniel Fekete. 2008. ZAME: Interactive Large-Scale Graph Visualization. In Proceedings of IEEE VGTC Pacific Visualization Symposium. 215-222.

Leonard Euler. 1741. Solutio problematis ad geometriam situs pertinentis. Commentari Academiae Scientiarum Petropolitanae 8 (1741), 128-140.

Thomas M. J. Fruchterman and Edward M. Reingold. 1991. Graph Drawing by Forcedirected Placement. Software, Practice, and Experience 21, 11 (1991), 1129-1164.

Mohammad Ghoniem, Jean-Daniel Fekete, and Philippe Castagliola. 2005. On the Readability of Graphs Using Node-Link and Matrix-Based Representations: A Controlled Experiment and Statistical Analysis. Information Visualization 4, 2 (2005), 114-135.

Martin Greilich, Michael Burch, and Stephan Diehl. 2009. Visualizing the Evolution of Compound Digraphs with TimeArcTrees. Computer Graphics Forum 28, 3 (2009), 975-982.

Nathalie Henry and Jean-Daniel Fekete. 2006. MatrixExplorer: a Dual-Representation System to Explore Social Networks. IEEE Transactions on Visualization and Computer Graphics 12, 5 (2006), 677-684.

Nathalie Henry and Jean-Daniel Fekete. 2007. MatLink: Enhanced Matrix Visualization for Analyzing Social Networks. In Proceedings of International Conference on Human-Computer Interaction INTERACT. 288-302.

Nathalie Henry, Jean-Daniel Fekete, and Michael J. McGuffin. 2007. NodeTrix: a Hybrid Visualization of Social Networks. IEEE Transactions on Visualization and Computer Graphics 13, 6 (2007), 1302-1309.

Danny Holten, Petra Isenberg, Jarke J. van Wijk, and Jean-Daniel Fekete. 2011. An Extended Evaluation of the Readability of Tapered, Animated, and Textured DirectedEdge Representations in Node-Link Graphs. In Proceedings of the IEEE Pacific Visualization Symposium, PacificVis. 195-202.

Michael Kaufmann and Dorothea Wagner (Eds.). 2001. Drawing Graphs, Methods and Models (the book grew out of a Dagstuhl Seminar, April 1999). Lecture Notes in Computer Science, Vol. 2025. Springer.

René Keller, Claudia M. Eckert, and P. John Clarkson. 2006. Matrices or NodeLink Diagrams: Which Visual Representation is Better for Visualising Connectivity Models? Information Visualization 5, 1 (2006), 62-76.

Mershack Okoe, Radu Jianu, and Stephen G. Kobourov. 2019. Node-Link or Adjacency Matrices: Old Question, New Insights. IEEE Transactions on Visualization and Computer Graphics 25, 10 (2019), 2940-2952.

Helen C. Purchase. 1997. Which Aesthetic has the Greatest Effect on Human Understanding?. In Proceedings of the International Symposium on Graph Drawing, GD. 248-261.

Ruth Rosenholtz, Yuanzhen Li, Jonathan Mansfield, and Zhenlan Jin. 2005. Feature Congestion: A Measure of Display Clutter. In Proceedings of the Conference on Human Factors in Computing Systems, CHI. 761-770.

Utkarsh Soni, Yafeng Lu, Brett Hansen, Helen C. Purchase, Stephen G. Kobourov, and Ross Maciejewski. 2018. The Perception of Graph Properties in Graph Layouts.
Computer Graphics Forum 37, 3 (2018), 169-181.

Tatiana von Landesberger, Arjan Kuijper, Tobias Schreck, Jörn Kohlhammer, Jarke J. van Wijk, Jean-Daniel Fekete, and Dieter W. Fellner. 2011. Visual Analysis of Large Graphs: State-of-the-Art and Future Research Challenges. Computer Graphics Forum 30, 6 (2011), 1719-1749.

Colin Ware. 2004. Information Visualization: Perception for Design. Morgan Kaufmann.

Colin Ware, Helen C. Purchase, Linda Colpoys, and Matthew McGill. 2002. Cognitive Measurements of Graph Aesthetics. Information Visualization 1, 2 (2002), 103-110. 